EPJ Web of Conferences 45, 01039 (2013)

DOI: $10.1051 /$ epjconf/20134501039

(C) Owned by the authors, published by EDP Sciences, 2013

\title{
Lorentz forces caused by rotating magnetic field
}

\author{
K. Horáková ${ }^{1}$ and K. Fraňa ${ }^{1}$ \\ ${ }^{1}$ Technical University of Liberec, Faculty of Mechanical Engineering, Department of Power Engineering Equipment, \\ Studentska 2, Liberec 1, Czech Republic
}

\begin{abstract}
Leading topic of this article is description of Lorentz forces in the container with cuboid shape. Inside of the container is an electrically conductive melt. Lorentz forces are caused by rotating magnetic field. Lorentz forces were calculated by analytic formula. This formula was derived for cylindrical container. Using this formula for cuboid container was assumed. Results were compared with Lorentz forces from CFD computing program NS-FEM3D. Areas of differences were recognized and displayed.
\end{abstract}

\section{Introduction}

A theory of flow behaviour under magnetic field effect is called magnetohydrodynamics (MHD).

First mentions about MHD appeared in relation to astrophysics and geophysics. Nowadays the magnetic field is used in technical practice. In a few papers were compared static and rotating magnetic fields [1]. The rotating magnetic field was turned out like better usable. Rotating magnetic field generates eddy flow in electric conductive melt. This effect is used to e.g. for noncontact electromagnetic stirring of the melt in metallurgy and crystal growing, when rotating magnetic field homogenize of varied metal alloy and fine metal. The melt flow positively affects metallographic structure of casts. More details about electromagnetic stirring are in [1].

A rotating magnetic field applied to an electrically conductive melt drives swirling flow caused by azimuthal Lorentz forces. Deduction of Lorentz forces analytical formula for cylindrical container was performed in [2]. In this article final analytical formula for cylindrical container will be transformed from cylindrical system of coordinates to Cartesian coordinate system and this formulation for Lorentz forces will be used for cuboid container. Lorentz forces for cuboid container from inhouse CFD code called NS-FEM3D will be displayed for comparing as well. Because of some simplifications in using analytical formula, areas of differences will be recognized and displayed.

\section{Analytical formula for Lorentz force}

The container is considered with electrically isolated walls, the melt inside the container is conductive with density $\rho$, kinematic viscosity $v$ and electric conductivity $\sigma$. Flow of the melt is driven by rotating magnetic field with magnetic induction $B$ (noted in the cylindrical coordinates):

$$
B=B_{0} \cdot \sin (\varphi-\varpi \cdot t) \cdot \mathbf{e}_{r}+B_{0} \cdot \cos (\varphi-\varpi \cdot t) \cdot \mathbf{e}_{\varphi} .
$$

In this formula (1) $\mathbf{e}_{r}$ and $\mathbf{e}_{\varphi}$ are unit vectors in radial and azimuthal direction and $\varpi$ is the constant angular frequency of the field.

Magnetic induction has only components $\mathrm{B}_{\mathrm{r}}$ a $\mathrm{B}_{\varphi}$ because it is assumed that vertical size of bipolar inductor is bigger than the height of the melt in the container. Vector potential $A$ was determined with $\mathbf{B}=\nabla \times \mathbf{A}=\operatorname{rot} \mathbf{A}$, thus:

$$
\mathbf{A}=-B_{0} \cdot r \cdot \cos (\varphi-\varpi \cdot t) \cdot \mathbf{e}_{\mathbf{z}} .
$$

The electric field intensity could be computed by this vector potential (2): $\mathbf{E}=-\left(\nabla \Phi_{\text {rot }}+\frac{\partial \mathbf{A}}{\partial t}\right)$, thus

$$
\mathbf{E}=-\nabla \Phi_{r o t}+B_{0} \cdot r \cdot \varpi \cdot \sin (\varphi-\varpi \cdot t) \cdot \mathbf{e}_{\mathbf{z}} .
$$

Non-dimensional frequency $K$ is defined:

$$
K=\mu \cdot \sigma \cdot \varpi \cdot R^{2}
$$

where $\mu$ is magnetic permeability, $\sigma$ is electric conductivity and $\varpi$ is magnetic field angular velocity. For $K<<1$ and force field is axisymmetric, the scalar potential $\Phi_{r o t}(r, \varphi, z, t)$ is possible to separate to two parts [3],

$$
\begin{aligned}
& \Phi_{r o t}(r, \varphi, z, t)=\Phi_{1}(r, z) \cdot \sin (\varphi-\varpi \cdot t) \\
& +\Phi_{2}(r, z) \cdot \cos (\varphi-\varpi \cdot t) .
\end{aligned}
$$

Force field is axisymmetric for cylindrical container. That condition is not valid for cuboid container. But scalar potential is not possible to deduce without this separation. Because of that, separation of scalar potential was performed for cuboid container as well. Incurred differential was under examination in the next part of this article. 
Current density $j$ is determined with

$$
\mathbf{j}=\sigma(\mathbf{E}+\mathbf{v} \times \mathbf{B}) \text {. }
$$

Because of low-induction and low-frequency it is possible to use some simplification for calculation current density $j$ and to use reduction of Ohm's law:

$$
\begin{aligned}
& \mathbf{j}=\sigma \cdot \mathbf{E} \Rightarrow \mathbf{j}=\sigma \cdot\left(-\frac{\partial \Phi_{r o t}}{\partial r} \cdot \mathbf{e}_{\mathbf{r}}-\frac{1}{r} \frac{\partial \Phi_{r o t}}{\partial \varphi} \cdot \mathbf{e}_{\varphi}\right. \\
& -\frac{\partial \Phi_{r o t}}{\partial z} \cdot \mathbf{e}_{z}+B_{0} \cdot r \cdot \varpi \cdot \sin (\varphi-\varpi \cdot t) \cdot \mathbf{e}_{\mathbf{z}}
\end{aligned}
$$

where $\sigma$ is the melt electric conductivity [1].

Using of equation $\nabla \cdot \mathbf{j}=\operatorname{div} \mathbf{j}=0$ is possible to get

$$
\text { equation: } \quad\left(\nabla^{2}-\frac{1}{r^{2}}\right) \cdot \Phi_{1}=0 \text {. }
$$

Solution of (8) is difficult because scalar potential $\Phi_{1}$ is bivariant function $(\mathrm{r}, \mathrm{z})$. Solution of $\Phi_{2}$ is after some computing zero. This equation (8) could be solved by the Fourier method of a variable separation and then solving two separate differential equations. The part which has the dependence only on the variable $r$ is converted to the shape of Bessel differential equations and then solved by Bessel functions. The second part which has the dependence only on the variable $\mathrm{z}$ is differential equation of the second order with constant coefficient and this equation is solved by the characteristic equation (for more details see to my earlier works [2]).

After some mathematical manipulations and solving boundary conditions the final analytical formula of scalar potential is:

$$
\begin{gathered}
\Phi(r, z)=\sum_{i=1}^{\infty} \frac{2 \cdot J_{1}\left(m_{i} \cdot r\right)}{m_{i} \cdot\left(m_{i}^{2}-1\right) \cdot J_{1}\left(m_{i}\right)} \\
\cdot \frac{\cosh \left(m_{i} \cdot z\right)-\cosh \left(m_{i} \cdot(H-z)\right)}{\sinh \left(m_{i} \cdot H\right)}
\end{gathered}
$$

where $m$ are roots of the equation $\mathrm{J}_{1}{ }^{\prime}(\mathrm{m})=0, z$ is nondimensional height of the container, $r$ is non-dimensional radius of the container, $H$ is non - dimensional total height of the container and $J_{1}$ is Bessel function of the first kind. This equation corresponds to the published results of other author see [4].

Lorentz forces are solved by formula:

$$
\mathbf{f}_{\text {rot }}=\mathbf{j}_{\text {rot }} \times \mathbf{B}_{\text {rot }} \text {. }
$$

After that Lorentz forces were time-averaged over one period $(2 \varpi)$. The final formula of time - averaged Lorentz forces in azimuthal direction is in this form:

$$
\begin{aligned}
& \overline{\mathbf{f}_{\text {rot } \varphi}}=\frac{\sigma \cdot B_{0}^{2} \cdot \varpi \cdot r}{2} \cdot\left(1-\frac{2}{r} \sum_{i=1}^{\infty} \frac{J_{1}\left(m_{i} \cdot r\right)}{\left(m_{i}{ }^{2}-1\right) \cdot J_{1}\left(m_{i}\right)}\right. \\
& \left.\frac{\sinh \left(m_{i} \cdot z\right)+\sinh \left(m_{i} \cdot(H-z)\right)}{\sinh \left(m_{i} \cdot H\right)}\right) .
\end{aligned}
$$

This equation corresponds to the published results of other authors as well, see [4, 5]. Forces in the radial and axial direction are zero in this simplification.

The conversion from cylindrical system of coordinates to Cartesian coordinate system is:

$$
\begin{aligned}
& f_{x}=f_{\varphi} \cdot \sin (\varphi)=-f_{\varphi} \cdot \frac{y}{\sqrt{x^{2}+y^{2}}} \\
& f_{y}=f_{\varphi} \cdot \cos (\varphi)=f_{\varphi} \cdot \frac{x}{\sqrt{x^{2}+y^{2}}} .
\end{aligned}
$$

After time-averaging over one period the final Lorentz forces are:

$$
\begin{gathered}
\overline{f_{x}}=-\frac{\sigma \cdot B_{0}^{2} \cdot \omega}{2} \cdot \sqrt{x^{2}+y^{2}} \cdot \frac{y}{\sqrt{x^{2}+y^{2}}} \\
+\frac{\sigma \cdot B_{0}^{2} \cdot \omega}{2} \cdot \frac{y}{\sqrt{x^{2}+y^{2}}} \\
\left.\cdot \sum_{i=1}^{\infty} \frac{2 \cdot J_{1}\left(m_{i} \cdot \sqrt{x^{2}+y^{2}}\right)}{\left(m_{i}^{2}-1\right) \cdot J_{1}\left(m_{i}\right)} \cdot \frac{\sinh \left(m_{i} \cdot z\right)+\sinh \left(m_{i} \cdot(H-z)\right)}{\sinh \left(m_{i} \cdot H\right)}\right) \\
\overline{f_{y}}=\frac{\sigma \cdot B_{0}^{2} \cdot \omega}{2} \cdot \sqrt{x^{2}+y^{2}} \cdot \frac{x}{\sqrt{x^{2}+y^{2}}}-\frac{\sigma \cdot B_{0}^{2} \cdot \omega}{2} \\
\frac{x}{\sqrt{x^{2}+y^{2}}} \cdot \sum_{i=1}^{\infty} \frac{2 \cdot J_{1}\left(m_{i} \cdot \sqrt{x^{2}+y^{2}}\right)}{\left(m_{i}^{2}-1\right) \cdot J_{1}\left(m_{i}\right)} \\
\left.\cdot \frac{\sinh \left(m_{i} \cdot z\right)+\sinh \left(m_{i} \cdot(H-z)\right)}{\sinh \left(m_{i} \cdot H\right)}\right) .
\end{gathered}
$$

Eq. 14 and 15 are valid for these conditions:

$x=0 \wedge y=0$ stand $\bar{f}_{x}=\bar{f}_{y}=0$.

$x \neq 0 \wedge y=0$ stand $\overline{f_{y}}=\overline{f_{\varphi}}, \quad \overline{f_{x}}=0$.

$x=0 \wedge y \neq 0$ stand $\bar{f}_{x}=\bar{f}_{\varphi}, \bar{f}_{y}=0$.

The final formula for Lorentz forces in Cartesian coordinate system is:

$$
\bar{f}=\sqrt{\left(\overline{f_{x}^{2}}+\overline{f_{y}^{2}}\right)} .
$$

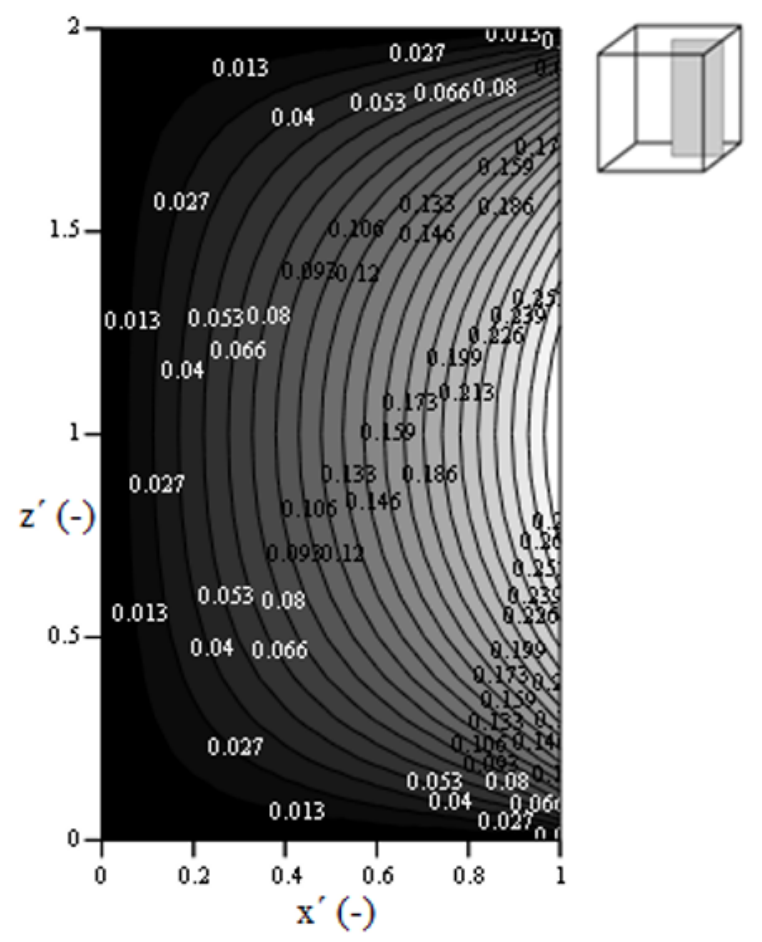

Fig. 1. Contours of time - averaged Lorentz forces in central plane (from analytical formula). 


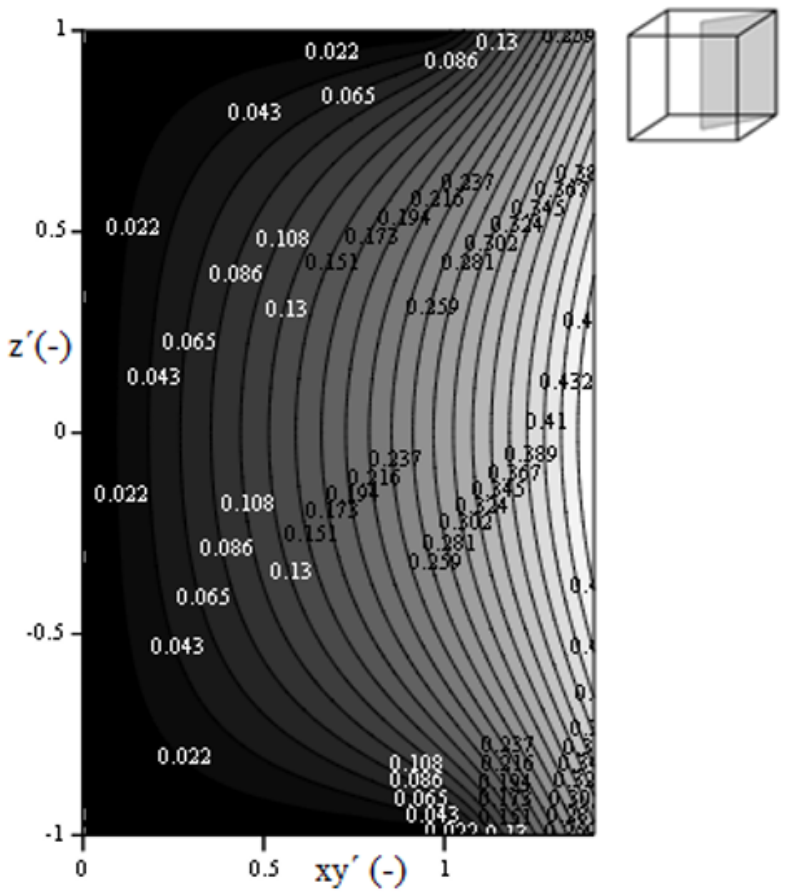

Fig. 2. Contours of time - averaged Lorentz forces in cross plane (from analytical formula).

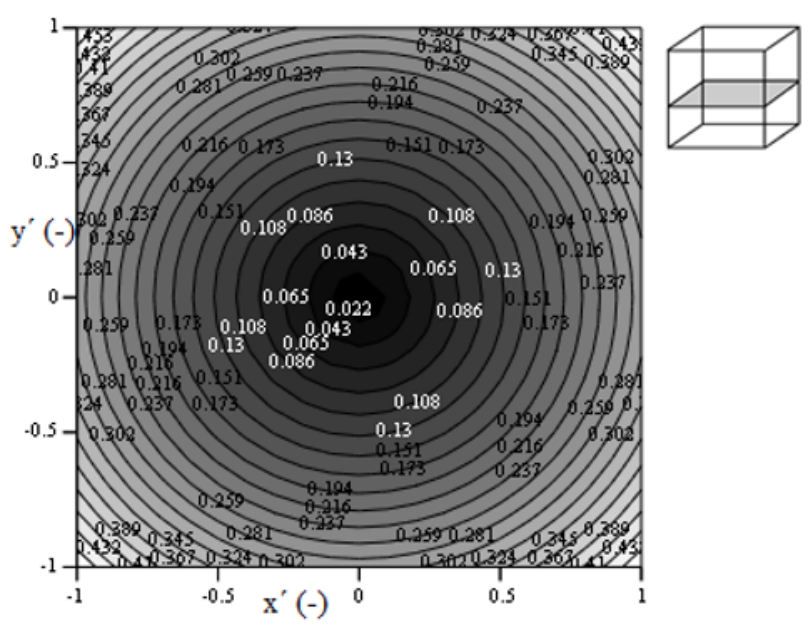

Fig. 3. Contours of time - averaged Lorentz forces in horizontal plane (from analytical formula).

Maxima of Lorentz forces are occurred in mid-plane with increasing distance from vertical axis of the container. Maximum values are detected near container vertical edges. Minimum values of Lorentz forces are appeared near vertical axis of the container. Lorentz forces near upper and lower bases should be minimal because of current density and magnetic induction are parallel. This condition is not performed in figure 2 because of simplification on equation 5 . In these areas are occurred maximum differences.

\section{Solutions in computing code NS- FEM3D}

Input data for comparing Lorentz forces in the container with cuboid shape were obtained from the computing program NS-FEM3D which uses DDES method of computing. The Delayed Detached Eddy Simulation model has been applied as a turbulent approach. This approach was implemented for higher Taylor number. Complete summary of applied mathematical model and validations see in [6]. The grid of container was unstructured. Whole grid has over 2200000 elements. Database from this computing code were created by data matrix - coordinates of grid node points, values of Lorentz forces component in the Cartesian coordinate system.

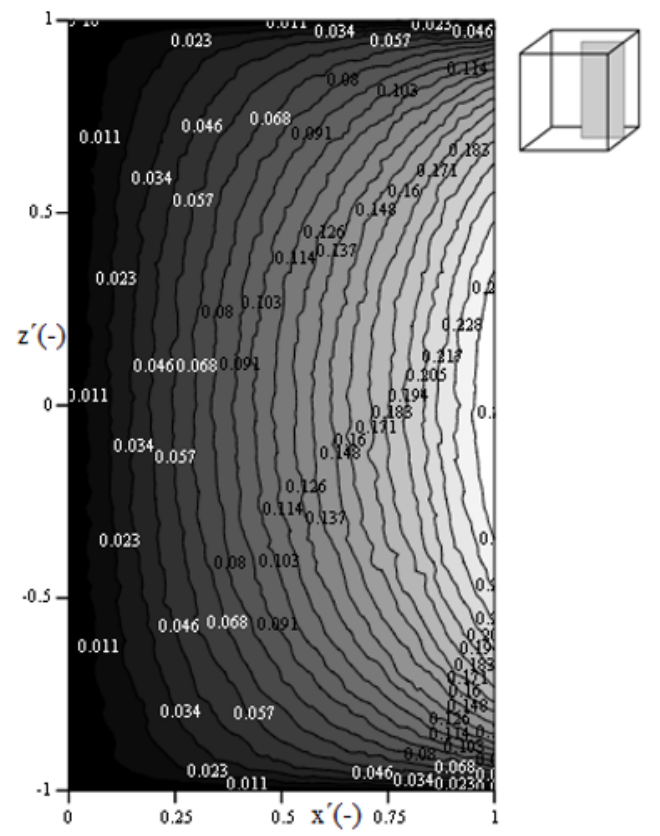

Fig. 4. Contours of time - averaged Lorentz forces in central plane (from NS-FEM3D).

And this database was processed in software MathCad (version 15). MathCad displayed maxima and minima (colour list) only for one figure. Colour list is different for each figure. Contours of Lorentz forces from this CFD code and analytical formula are very similar. Maximum values of Lorentz forces are detected near container vertical edges. Minimum values of Lorentz forces are appeared near vertical axis of the container and lower and upper bases.

\section{Comparing results}

Comparing Lorentz forces from analytical formula and CFD code NS-FEM3D it can be see relative agreement. The best correspondence is shown in volume of insert cylinder to this cuboid. Good agreement is in the area of solid line, dash line indicate bigger differences. Forces from analytical formula are slightly bigger. In areas of vertical edges are occurred maximum differences because of simplification on equation 5 . 


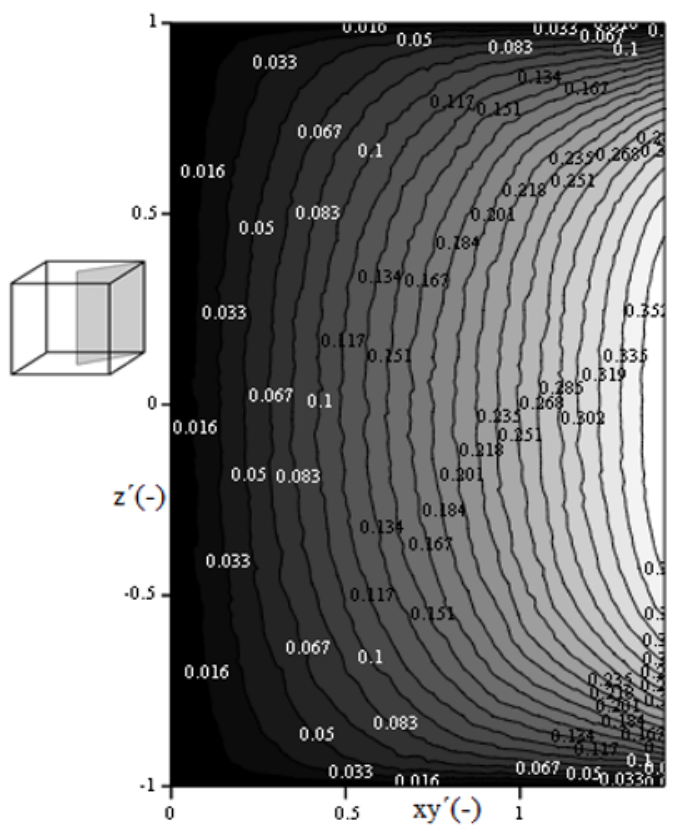

Fig. 5. Contours of time - averaged Lorentz forces in cross plane (from NS-FEM3D).
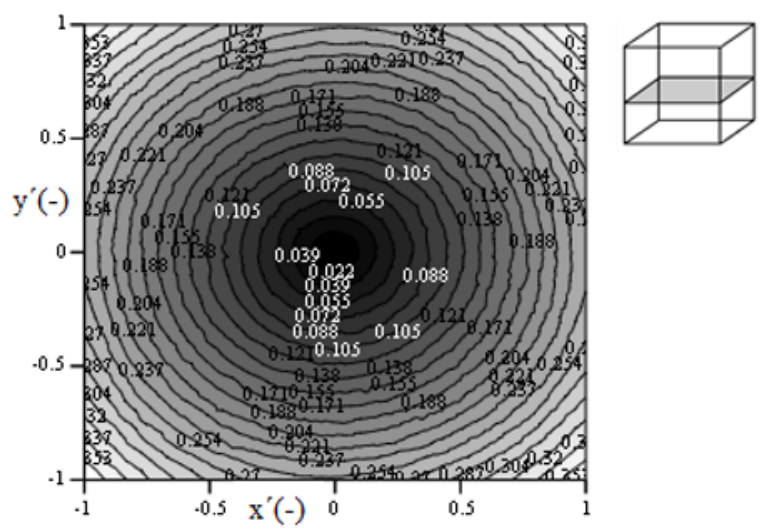

Fig. 6. Contours of time - averaged Lorentz in horizontal plane (from NS-FEM3D).

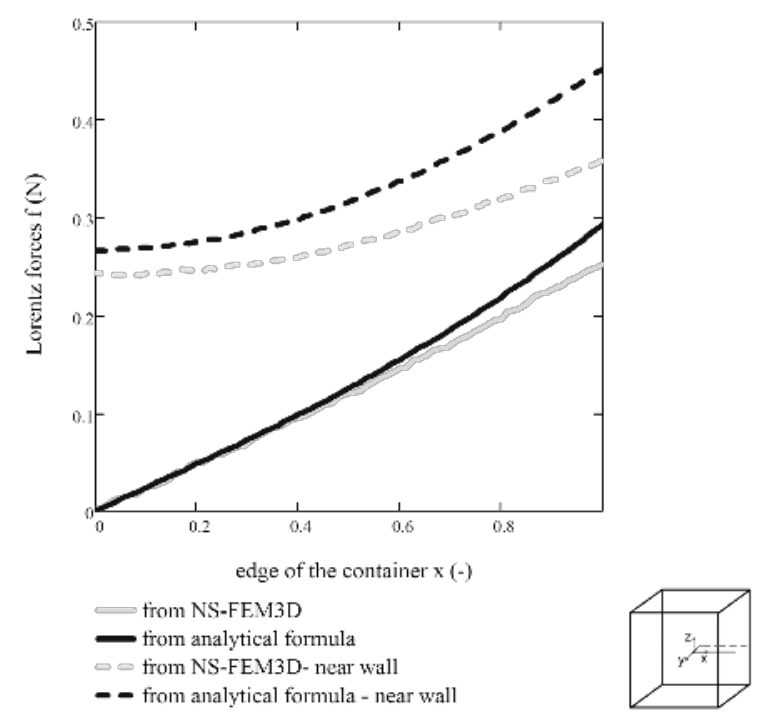

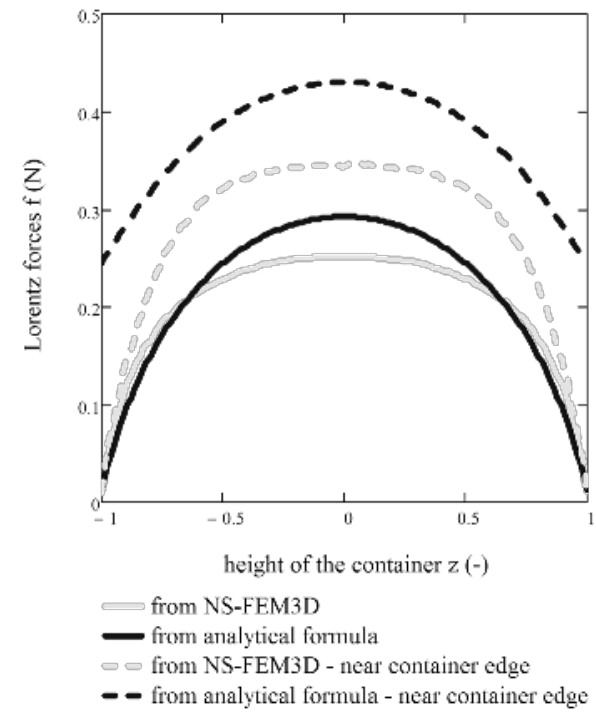

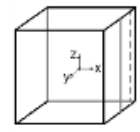

Fig. 8. Comparing values of Lorentz forces from analytical formula and NS-FEM3D in dependence on container height.

\section{Conclusions}

Deduction of Lorentz forces analytical formula for cuboid container was performed in this article. This formula was obtained by transformation of analytical formula from cylindrical container. Contours of Lorentz forces obtained from CFD code NS-FEM3D and analytical formula were displayed. A simplification in deduction caused differences. Minimum differences are appeared in area of mid-plane. Maximum of these differences are occurred in area of vertical container edges. In these areas damping function should be used. That is scope of the next work.

\section{Acknowledgements}

This work was financially supported by the particular research student grant SGS 2823 at TU of Liberec.

\section{References}

1. P. A. Davidson, An Introduction to magnetohydrodynamics, (2001)

2. K. Horáková, K. Fraňa, JASTFM, 2 (2009)

3. J. Priede, Theoretical study of a flow in an axisymetric cavity of finite length, driven by a rotating magnetic field (1993)

4. Marty PH, M. Witkowski L., Transfer Phenomena in Magnetohydrodynamic and Electroconduction Flows, pp. 327-343 (1999)

5. P. A. Nikrityuk, K. Eckert, R. Grundmann, Metallurgical and Materials Transactions B 37 (2006)

6. J. Stiller, K. Fraňa, A. Cramer, J. Phys. Fluids, 18 (2006)
Fig. 7. Comparing values of Lorentz forces from analytical formula and NS-FEM3D in dependence on container edge. 